\title{
The Level of Confidence, Readiness and its Associated Factors Among Medical Graduates Prior to House Officer (HO) Training
}

\author{
Aneesa Abdul Rashid ${ }^{1}$,Sazlina Shariff Ghazali ${ }^{1}$, Iliana Mohamad ${ }^{2}$, Dalila \\ Roslan $^{3}$, Husna Musa ${ }^{4}$, Maliza Mawardi ${ }^{1}$ \\ ${ }^{1}$ Department of Family Medicine, Faculty of Medicine and Health Sciences, University Putra Malaysia, \\ Serdang, Malaysia; ${ }^{2}$ Medigrow (Medicorp Resources), Batu Caves, Selangor, Malaysia; ${ }^{3}$ Kuala Pilah \\ Health District Office, Ministry of Health Malaysia, Negeri Sembilan, Malaysia; ${ }^{4}$ Department of \\ Paediatrics, Faculty of Medicine and Health Sciences, University Putra Malaysia, Serdang, Malaysia
}

\begin{abstract}
Introduction: House officers are reported to feel less confident in working, especially in the initial part of their training. Among the factors that make them feel unprepared are in terms of adequate knowledge, clinical skills and dealing with workload. The impact of less confident house officers $(\mathrm{HO})$ can lead to detrimental effects.
\end{abstract}

Objectives: This study aims to look into the confidence and readiness levels of medical graduates prior to starting a $\mathrm{HO}$ preparatory course and factors associate.

Methods: This is a cross sectional questionnaire study done among medical graduates between April - September 2018. This is part of a bigger study looking into the effectiveness of this course, which was initially organised under the Islamic Medical Association of Malaysia (IMAM) and later privatized to Medicorp (a medical training company for junior doctors). 238 participants who fulfilled the inclusion and exclusion criteria, completed the adapted IMU Competency survey pre and post the course. Their level of confidence and readiness to work was scored out of a Likert scale of 1 to 5. The higher the score, the higher the confidence or readiness to work.

Results: We found significant association of confidence levels pre-course with ethnicity $(\mathrm{p}=0.038)$. The scores were $2.0(\mathrm{IQR}=2.0), 2.0(\mathrm{IQR}=2.0), 3.0(\mathrm{IQR}=1.0), 2.5(\mathrm{IQR}=1.25)$ for Malay, Chinese, Indian and others respectively. We report significant associations with level of confidence $(p=0.03)$ and readiness $(p=0.08)$ not only prior, but levels of confidence $(p<0.001)$ and readiness $(p<0.001)$ after the course in association with place of study. Significant association was found for levels of confidence $(p=0.048)$ and readiness $(p=0.07)$ according to gender for scores post-course which were both $3.0(\mathrm{IQR}=1.0)$ and $4.0(\mathrm{IQR}=1.0)$ for females and males respectively.

Conclusion: Confidence level to start work were higher for the Indian ethnicity before the HOpreparatory-course. Confidence and readiness levels were higher in male after the course, and locally graduated participants pre and post course.

Keywords: house officer; psychological; wellbeing; confidence; readiness 